\title{
CORE CURVES OF TRIANGULATED SOLID TORI
}

\author{
MARC LACKENBY
}

\begin{abstract}
We show that in any triangulation of a solid torus there is a precore curve that lies in the 2-skeleton and that intersects the interior of each face in at most 10 straight arcs. By definition, a pre-core curve is a simple closed curve that becomes a core curve when a collar is attached to the boundary of the solid torus. This theorem imposes restrictions on the possible Riemannian metrics on a solid torus. It also has applications in knot theory.
\end{abstract}

\section{INTRODUCTION}

One of the most powerful tools in 3-manifold topology is normal surface theory. Many topologically relevant surfaces can be placed into normal form (or some variant of this) with respect to any triangulation $T$ of the manifold $M$. An important case of this phenomenon is when $M$ is the solid torus and the surface is a meridian disc. The existence of a meridian disc in normal form and the fact that this can be algorithmically detected is the key to Haken's solution to the problem of recognizing the unknot [1. However, normal surface theory suffers from some substantial limitations, possibly the most important of which is that every normal meridian disc may have 'exponential complexity'. More precisely, if $n$ is the number of tetrahedra in $T$, then normal surface theory only produces a meridian disc $D$ with at most $2^{k n}$ normal triangles and squares, where $k=10^{11}$ (see [2]). This is more than just an artifice of the theory, because one can find triangulations of the solid torus where every normal meridian disc has exponentially many squares and triangles. Indeed, we will do this explicitly in Section 6. However, in this paper, we show that for a related problem there is a solution with linearly bounded complexity. In addition to the meridian disc, the solid torus contains another important sub-object: the core curve $C$, which is defined, up to ambient isotopy, to be $\{*\} \times S^{1} \subset D^{2} \times S^{1}$, where $*$ is a point in the interior of $D^{2}$. In this paper, we will address the problem of placing $C$ into 'normal form'. The surprising conclusion is that this can be achieved with a linear upper bound on the 'complexity' of $C$.

In order to state our main result most cleanly, we say that a curve $C$ embedded in the solid torus $M$ is a pre-core curve if $C$ is a core curve in the manifold $M \cup(\partial M \times I)$ that is obtained from $M$ by attaching a collar to $\partial M$. Thus, a pre-core curve need not lie in the interior of $M$.

Whenever we refer to a triangulation of a 3-manifold $M$, we use the more general definition that is now standard in low-dimensional topology. Thus, it is an expression of $M$ as a collection of 3-simplices, with some of their faces identified in pairs, via affine homeomorphisms. Since the gluing maps are affine, it makes sense to speak of a straight line in a face of the triangulation.

Received by the editors February 2, 2012 and, in revised form, March 19, 2013.

2010 Mathematics Subject Classification. Primary 57N10.

(C) 2014 by the author 
The following is our main result.

Theorem 1.1. Let $T$ be a triangulation of the solid torus $M$. Then $M$ contains a pre-core curve $C$ that lies in the 2-skeleton of $T$, intersects the edges in finitely many points, is disjoint from the vertices and intersects the interior of each face in at most 10 straight arcs.

Note that this does not bound the number of intersection points between $C$ and each edge, face or tetrahedron of $T$. This is because as $C$ runs from one face to another, the result is a point of intersection with an edge of $T$, and this may give rise to isolated points of intersection with the faces and tetrahedra incident to that edge. If one wants to bound the total number of points of intersection between a core curve and each tetrahedron, it seems to be best to make the curve transverse to the 2-skeleton of $T$, as follows.

Theorem 1.2. Let $T$ be a triangulation of the solid torus $M$. Then $M$ contains a core curve $C^{\prime}$ that intersects each tetrahedron $\Delta$ of $T$ in at most 18 arcs. Moreover, each such arc is properly embedded in $\Delta$ and has endpoints in the interior of the faces of $\Delta$. The intersection $\Delta \cap C^{\prime}$ is a trivial tangle in $\Delta$. In fact, $\Delta \cap C^{\prime}$ is parallel to a collection of arcs $\alpha$ in $\partial \Delta$, with the property that the intersection between each component of $\alpha$ and each face of $\Delta$ is at most one straight arc.

Thus, the above result asserts that there is a core curve that intersects each tetrahedron in one of finitely many possible trivial tangles, where this finite list is universal, in the sense that it is independent of the triangulation $T$.

In addition, we will prove similar theorems for partially ideal triangulations of the solid torus, and for 'affine' handle structures. (See Theorems 4.1 and 4.2.)

Although Theorem 1.1 is a result about triangulations, it has geometric consequences. The reason is that a triangulation of a manifold $M$ determines a path metric in which each tetrahedron is regular and Euclidean, and, conversely, any Riemannian metric can be approximated (in a suitable sense) by such a piecewise Euclidean metric. By using this relationship between triangulations and Riemannian metrics, and applying Theorem 1.1, we obtain the following result.

Theorem 1.3. For each $K, I>0$, there is a constant $c(K, I)$ with the following property. If $M$ is a solid torus with a Riemannian metric having volume at most $V$, injectivity radius at least $I$ and all sectional curvatures in the interval $(-K, K)$, then $M$ contains a core curve with length at most $c(K, I) V$.

It seems likely that Theorems 1.1 and 1.2 will have many other applications. In fact, the version of these theorems for affine handle structures is a key step in the proof of the main theorem in 7 . This asserts that the crossing number of a satellite knot is at least $10^{-13}$ times the crossing number of its companion knot. For more details and an explanation of this terminology, see [7.

We now give an outline of the proof of Theorems 1.1 and 1.2. We may find a meridian disc $D$ for the solid torus $M$ that is normal with respect to the triangulation $T$. If we cut $M$ along $D$, the result is a 3 -ball $X$. The parts of $D$ lying between adjacent parallel normal discs patch up to form an $I$-bundle in $X$, which we term the parallelity bundle $\mathcal{B}$. (A precise definition is given in Section 2.) Now, in each 3 -simplex $\Delta$ of $T$, at most 6 components of $\Delta-N(D)$ do not lie in $\mathcal{B}$. The goal, therefore, is to find a core curve $C$ that avoids $\mathcal{B}$ and that intersects each component of $\Delta-N(D)$ in a bounded number of arcs with controlled topology. However, 


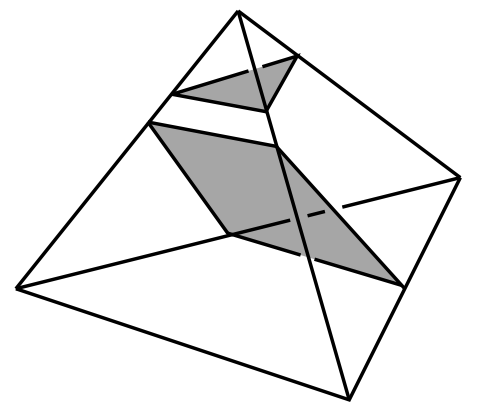

Figure 1

this is not completely straightforward. For example, $\mathcal{B}$ may be almost all of $X$, and so it is not clear that there is even a single core curve disjoint from $\mathcal{B}$, particularly one with controlled intersection with each tetrahedron. The technique that we use is to construct a product structure $D^{2} \times[-1,1]$ on $X$, where $D^{2} \times\{-1,1\}$ is the copies of $D$ in $X$, and with the property that the product structure agrees with the $I$-bundle structure on (most components of) $\mathcal{B}$. This product structure determines a homeomorphism $\phi: D^{2} \times\{1\} \rightarrow D^{2} \times\{-1\}$. We also have a homeomorphism $\psi: D^{2} \times\{-1\} \rightarrow D^{2} \times\{1\}$ arising from the gluing map. Their composition $\psi \phi$ is a homeomorphism $D^{2} \times\{1\} \rightarrow D^{2} \times\{1\}$. By the Brouwer fixed point theorem, this has a fixed point $x \in D^{2} \times\{1\}$. There are essentially two cases: either $x$ lies in $\mathcal{B}$ or it does not. If it does, then $x \times[-1,1]$ is a vertical curve in $\mathcal{B}$, which therefore joins adjacent parallel normal discs of $D$. But the two endpoints of $x \times[-1,1]$ are identified under the gluing map, because $x$ is a fixed point of $\psi \phi$, which is a contradiction. Thus, $x$ does not lie in $\mathcal{B}$, and with some more work, one can arrange that (a copy of) $x \times[-1,1]$ avoids $\mathcal{B}$. The endpoints of this arc patch together to form the required core curve of $M$.

\section{Normal SURfaces AND PARALlELITy BUNDLES}

In this section, we recall some basic normal surface theory and then go on to define parallelity bundles.

Recall that a disc properly embedded in a 3 -simplex $\Delta$ is normal if it is disjoint from the vertices, it intersects each edge transversely in at most one point, but it is not disjoint from the edges. It is then a triangle or square, as shown in Figure 1. A properly embedded surface in a triangulated 3-manifold is normal if it intersects each 3-simplex of the triangulation in a collection of normal discs.

Any normal surface may be straightened with respect to the triangulation, as follows. First ambient isotope the arcs of intersection with the faces, so that they become straight. Each triangle in each 3-simplex may then be ambient isotoped, keeping its boundary fixed, so that it becomes planar in the affine structure. The same is not necessarily true of the squares. But we may realise each square as the union of two flat pieces glued along a straight line. We will henceforth assume that any normal surface has been straightened in this way.

An arc properly embedded in a 2-simplex is normal if it is disjoint from the vertices and has endpoints lying in distinct edges. If $F$ is a compact surface with a triangulation, then a 1-manifold properly embedded in $F$ is normal if it intersects 
each 2-simplex in a collection of normal arcs. Define the length of a normal 1manifold in $F$ to be its number of intersections with the 1-skeleton.

The following is variant of a well-known fact in normal surface theory. (See Proposition 4.4 in [6] for comparison.)

Proposition 2.1. Let $T$ be a triangulation of a compact irreducible 3-manifold $M$. Let $S$ be a properly embedded, oriented, incompressible surface in $M$, with no components that are 2-spheres or boundary-parallel discs. Give $\partial S$ the orientation that it inherits from $S$. Suppose that $\partial S$ does not intersect any edge of $T \cap \partial M$ in two points with opposite orientations. Then, there is an ambient isotopy, keeping $\partial S$ fixed, taking $S$ into normal form.

Proof. In the usual normalisation procedure, there is one step where $\partial S$ may need to be moved. This happens as follows. Suppose that there is an arc of intersection between $S$ and a face of $T$, with endpoints in the same edge of the face, and suppose that this edge lies in $\partial M$. The standard way to argue is to assume that $S$ is boundary-incompressible and that $\partial M$ is incompressible, and thence perform an isotopy which removes the arc. However, we do not follow this line of argument here. Instead, we note that at the endpoints of the arc, $\partial S$ intersects the edge with opposite signs, which is contrary to the assumption. Thus, $\partial S$ does not need to be moved.

Corollary 2.2. Let $M$ be a solid torus with a triangulation $T$. Let $\gamma$ be a normal curve in $\partial M$ that bounds a meridian disc. Suppose that $\gamma$ has shortest length among all such curves. Then $\gamma$ bounds a normal meridian disc.

Proof. Suppose that $\gamma$ intersects some edge of $\partial M$ in two points of opposite sign. We may assume that these two points of intersection are adjacent on the edge. Let $\rho$ be the sub-arc of the edge between then. Then, the interior of $\rho$ lies in the annulus $\partial M-\gamma$. By our assumption about orientations, $\rho$ is inessential in this annulus. Hence, a sub-arc of $\gamma$ is parallel in $\partial M$ to $\rho$. We may isotope this sub-arc onto $\rho$, and then reduce its length. This is contrary to the hypothesis. So, the conditions of Proposition 2.1 are satisfied, and hence $\gamma$ bounds a normal meridian disc.

We will now give the definition of a parallelity bundle. Let $M$ be a compact orientable 3-manifold with a triangulation $T$. Let $S$ be a properly embedded normal surface in $M$, and let $X$ be the manifold obtained by cutting $M$ along $S$. In other words, $X$ is the closure of $M-N(S)$. Thicken the simplices of $T$ into handles, forming a handle structure $\widehat{\mathcal{H}}$. Thus, each $i$-simplex of $T$ becomes an $i$-handle. Then $S$ intersects each handle of $\widehat{\mathcal{H}}$ in a collection of discs. We say that two such discs are of the same type if there is an ambient isotopy of $M$, preserving all the handles, that takes one disc to the other. If $D_{1}$ and $D_{2}$ are adjacent discs of the same type in a handle $H$, then the component of $H-\operatorname{int}\left(N\left(D_{1} \cup D_{2}\right)\right)$ that lies between them is a product region $D^{2} \times I$, where $D^{2} \times \partial I$ is parallel to $D_{1} \cup D_{2}$. The union of these product regions is the parallelity bundle $\mathcal{B}$ in $X$. (See Figure 2.) The product structures patch together to form an $I$-bundle structure on $\mathcal{B}$. Its horizontal boundary $\partial_{h} \mathcal{B}$ is the $\partial I$-bundle, and it lies in $\partial N(S)$. The vertical boundary $\partial_{v} \mathcal{B}$ is $\operatorname{cl}\left(\partial \mathcal{B}-\partial_{h} \mathcal{B}\right)$. The vertical boundary $\partial_{v} \mathcal{B}$ of $\mathcal{B}$ is a collection of annuli. The intersection of $\partial_{v} \mathcal{B}$ with $\partial M$ is a union of fibres in the $I$-bundle structure. This is because this is true in each product region $D^{2} \times I$ that makes up $\mathcal{B}$. 

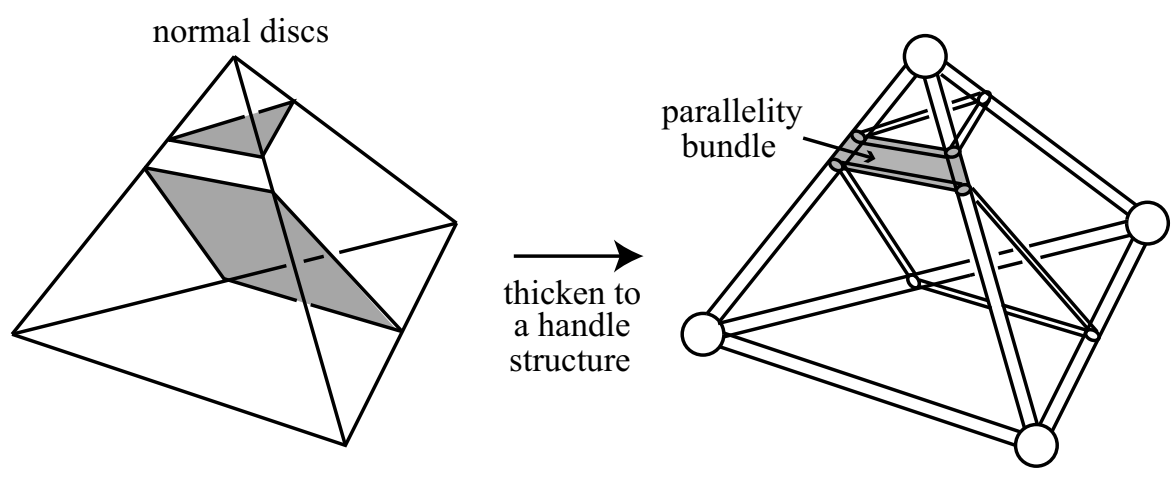

FiguRe 2

\section{Proof of the MAIN THEOREMS}

In this section, we prove Theorems 1.1 and 1.2.

Proof. Let $D$ be a properly embedded meridian disc, in normal form with respect to $T$. Define the weight of $D$ to be the number of points of intersection between $D$ and the 1-skeleton of $T$. Define the length of $\partial D$ to be its number of points of intersection with the 1-skeleton. We define the complexity of $D$ to be an ordered pair of non-negative integers, the first of which is the length of $\partial D$ and the second of which is the weight of $D$. We order these pairs lexicographically, which is a wellordering, and choose $D$ so that it has minimal complexity. Note that this definition of complexity is a little different from most treatments of the subject, where the weight of $D$ is given more significance than the length of $\partial D$. (See [3] and [8] for example.) However, this alternative definition of complexity is more suited to our purposes.

Note that $\partial D$ is a shortest normal curve in $\partial M$ that bounds a meridian disc. For suppose that there were a shorter normal meridian curve. By Corollary 2.2, this would bound a normal meridian disc, which would therefore have smaller complexity than $D$, which is a contradiction.

Let $N(D)$ be a thin regular neighbourhood of $D$ consisting of a union of normally parallel copies of $D$. Let $X$ be the result of removing $\operatorname{int}(N(D))$ and $\operatorname{int}(N(D) \cap \partial M)$ from $M$. Thus, $X$ is a 3 -ball, with two copies of $D$ in its boundary, which we denote by $D_{-}$and $D_{+}$. Let $A$ be the annulus $\partial X-\operatorname{int}\left(D_{-} \cup D_{+}\right)=\operatorname{cl}(\partial M-N(D))$.

Thicken each $i$-simplex of $T$ to an $i$-handle, forming a handle structure $\widehat{\mathcal{H}}$ for $M$. Let $\mathcal{B}$ be the parallelity bundle for $X$, as described in Section 2. Let $\mathcal{B}^{\prime}$ be the union of those components of $\mathcal{B}$ that intersect $A$. Some possible configurations for components of $\mathcal{B}$ are shown in Figure 3. Only the top two lie in $\mathcal{B}^{\prime}$.

We are now in a position to give a more detailed overview of the proof of Theorems 1.1 and 1.2. Our main goal in this proof is to construct an unknotted arc in $X$ with one endpoint in $D_{-}$and the other in $D_{+}$, so that these endpoints are identified when $D_{-}$and $D_{+}$are glued together to form $M$. In order to gain sufficient control over the arc, it will be important that it avoids $\mathcal{B}$. We therefore need to understand the topology of $X-\mathcal{B}$. As indicated in Figure 3, the components of $\mathcal{B}-\mathcal{B}^{\prime}$ can be embedded in $X$ in a topologically complicated way. However, we will show in Claims $1-3$ that $\mathcal{B}^{\prime}$ is in fact a product $I$-bundle, and the product structure on 

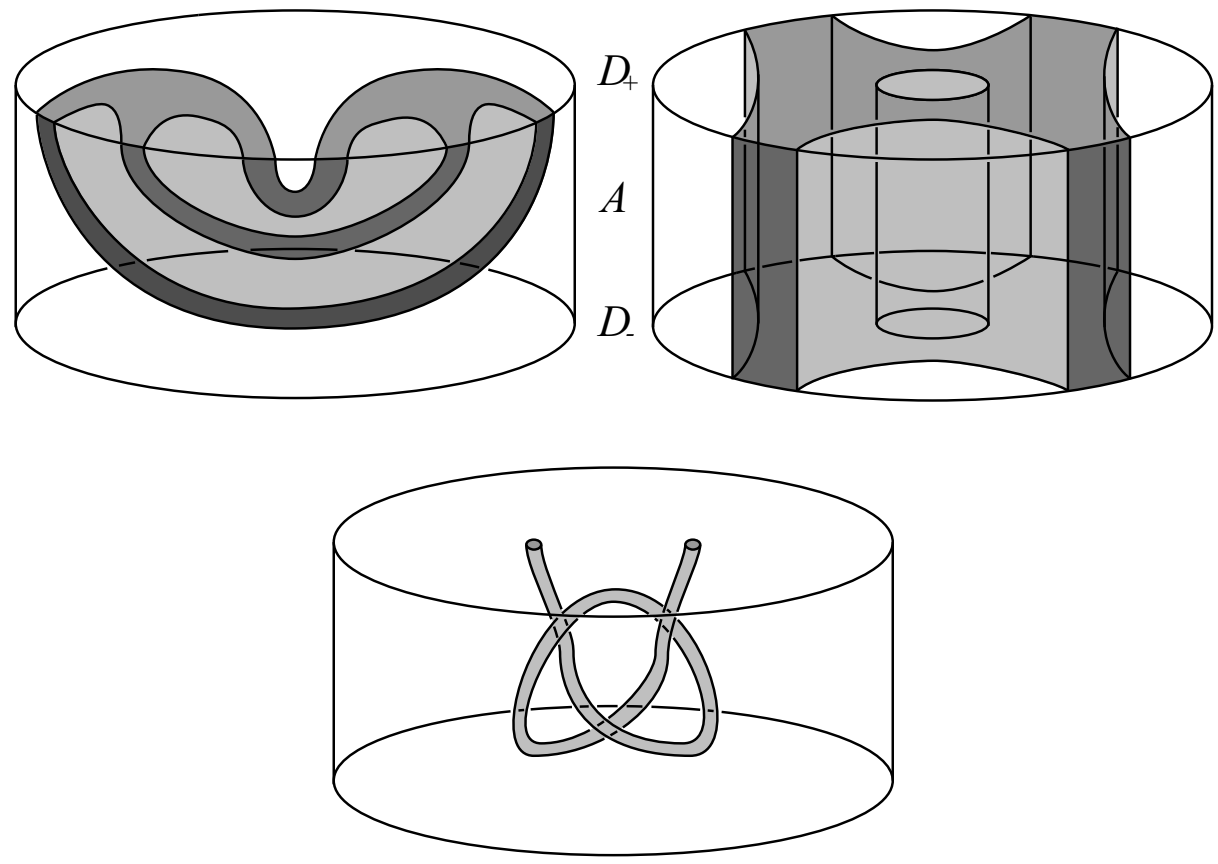

FiguRE 3

$X$ can be chosen so that it restricts to the product structure on $\mathcal{B}^{\prime}$. The product structure on $X$ determines a homeomorphism from $D_{+}$to $D_{-}$. If we compose this with the gluing map, we obtain a homeomorphism $D_{+} \rightarrow D_{+}$. By Brouwer's theorem, this has a fixed point $x$. In Claim 4 , we show that this homeomorphism may be chosen so that $x$ and its image in $D_{-}$are disjoint from $\mathcal{B}-\mathcal{B}^{\prime}$. Thus, there are three possibilities:

1. $x \in \mathcal{B}^{\prime}$

2. $x$ lies in a component of $D_{+}-\mathcal{B}^{\prime}$ that has non-empty intersection with $\partial D_{+}$;

3. $x$ lies in a component of $D_{+}-\mathcal{B}^{\prime}$ that is disjoint from $\partial D_{+}$.

Case 1 leads to an immediate contradiction. Case 2 forms the most substantial part of the argument. In this situation, we construct the required $\operatorname{arc}$ in $X$, by first running from $x$ in $D_{+}-\mathcal{B}$ to $\partial D_{+}$, then along $A$, and then in $D_{-}-\mathcal{B}$ to the image of $x$ under the gluing map. In Case 3 , we focus on the component $R_{+}$ of $\operatorname{cl}\left(D_{+}-\mathcal{B}^{\prime}\right)$ that contains $x$. If $\partial R_{+}$has non-trivial intersection with its image under the homeomorphism $D_{+} \rightarrow D_{+}$, then we may construct the required arc in a similar way to Case 2. However, instead of running along $A$, it runs along a portion of the vertical boundary of $\mathcal{B}^{\prime}$. On the other hand, if $\partial R_{+}$is disjoint from its image, we reach a contradiction by showing that the initial meridian disc $D$ did not have minimal complexity.

We now embark upon this proof.

Claim 1. The $I$-bundle structure on $\mathcal{B}$ is in fact a product structure. 
Now, $\mathcal{B}$ is an $I$-bundle over a surface $F$, and to assert that $\mathcal{B}$ is a product is equivalent to asserting that $F$ is orientable, because the total space of $\mathcal{B}$ is orientable. Suppose therefore that some component $F^{\prime}$ of $F$ is non-orientable. Let $E$ be the corresponding component of $\mathcal{B}$. Then the horizontal boundary of $E$ is connected. Double the 3-ball $X$ along $A$ to form a copy of $S^{2} \times I$. Glue the two copies of $E$ in $S^{2} \times I$ together to form a bundle $E^{+}$in $S^{2} \times I$. Now, $E^{+}$need not be connected (because $E$ may be disjoint from $A$ ), but if it is not, discard one of its components. Because $E \cap A$ is a union of fibres, $E^{+}$is an $I$-bundle over a surface $F^{+}$. Note that $F^{+}$is non-orientable, since we are assuming that $F^{\prime}$ is non-orientable. Hence, the horizontal boundary of $E^{+}$is connected. It is a planar surface lying in one component of $S^{2} \times \partial I$. Each of its boundary components therefore bounds a disc with interior disjoint from $E^{+}$. We can view $F^{+}$as the zero section of the $I$-bundle $E^{+}$. Each of its boundary components is a core curve of an annular component of $\partial_{v} E^{+}$. It is therefore parallel to a boundary component of this annulus, which in turn bounds a disc in $S^{2} \times \partial I$. Thus, we may extend $F^{+}$to form a closed embedded non-orientable surface in $S^{2} \times I$. Then attaching 3-balls, we get such a surface in $S^{3}$. But it is well known that $S^{3}$ contains no such surface. This proves the claim.

Claim 2. Each component of $\mathcal{B}^{\prime}$ must intersect both $D_{-}$and $D_{+}$.

Suppose that, on the contrary, some component $E$ of $\mathcal{B}^{\prime}$ is disjoint from $D_{-}$or $D_{+}$, as in the top left of Figure 3, for example. Then each fibre of $E \cap A$ is inessential in $A$. So some component of $\operatorname{cl}(A-E)$ is a disc $P$, with boundary consisting of an $\operatorname{arc} \alpha$ in $\partial A$ together with an $I$-fibre $\beta$ in $\partial_{v} \mathcal{B}^{\prime}$. Since $\beta$ is disjoint from the 1-skeleton of $T$, it therefore has zero length. Now, $\alpha$ is parallel to a sub-arc $\alpha^{\prime}$ in $\partial D$. This has positive length, because the endpoints of $\beta$ lie in distinct normal discs of $D$. We can attach $P$ to $D$, forming a new meridian disc for the solid torus $M$. This has the effect of replacing $\alpha^{\prime}$ with $\beta$, which reduces the length of $\partial D$, and this is a contradiction.

It is a consequence of Claim 2 that each fibre of $\mathcal{B}^{\prime} \cap A$ is essential in $A$ (and this is in fact what is proved). For suppose some fibre of $\mathcal{B}^{\prime} \cap A$ were inessential in $A$. Then the endpoints of this arc would both lie in $D_{-}$or would both lie in $D_{+}$. Since this arc joins the two parts of the horizontal boundary of this component of $\mathcal{B}^{\prime}$, we deduce that this component of $\mathcal{B}^{\prime}$ is disjoint from $D_{-}$or $D_{+}$, contradicting Claim 2.

Claim 3. We may pick a product structure $D \times[-1,1]$ on $X$ so that $D_{-}=D \times\{-1\}$, $D_{+}=D \times\{1\}$, and so that the product structure agrees with that on $\mathcal{B}^{\prime}$.

Let $S_{+}$and $S_{-}$be the surfaces $D_{+} \cap \mathcal{B}^{\prime}$ and $D_{-} \cap \mathcal{B}^{\prime}$. Pick a maximal collection $\alpha$ of disjoint non-parallel arcs properly embedded in $S_{+}$that are each essential in $S_{+}$and that have boundary lying in $\partial D_{+}$. Because each component of $\mathcal{B}^{\prime}$ intersects $\partial D_{+}$, by the definition of $\mathcal{B}^{\prime}$, each component of $S_{+}-\alpha$ is therefore a disc or an annulus. (Note that $\alpha$ may be empty if $S_{+}$is already a union of discs and annuli.) Let $\alpha^{\prime}$ be the arc components of $\operatorname{cl}\left(\partial S_{+}-\partial D_{+}\right)$. Using the product structure on $\mathcal{B}^{\prime}$, which exists by Claim 1, pick a collection of embedded vertical discs $V$ in $\mathcal{B}^{\prime}$ such that $V \cap D_{+}=\alpha \cup \alpha^{\prime}$, and which are properly embedded in $X$. Each such disc has boundary consisting of an $\operatorname{arc}$ in $D_{+}$, an $\operatorname{arc}$ in $D_{-}$and two $\operatorname{arcs}$ in $A$, by Claim 2. Cut $X$ along these discs, giving a collection of balls. We will now pick a product structure on these balls, which will patch together to give the required 


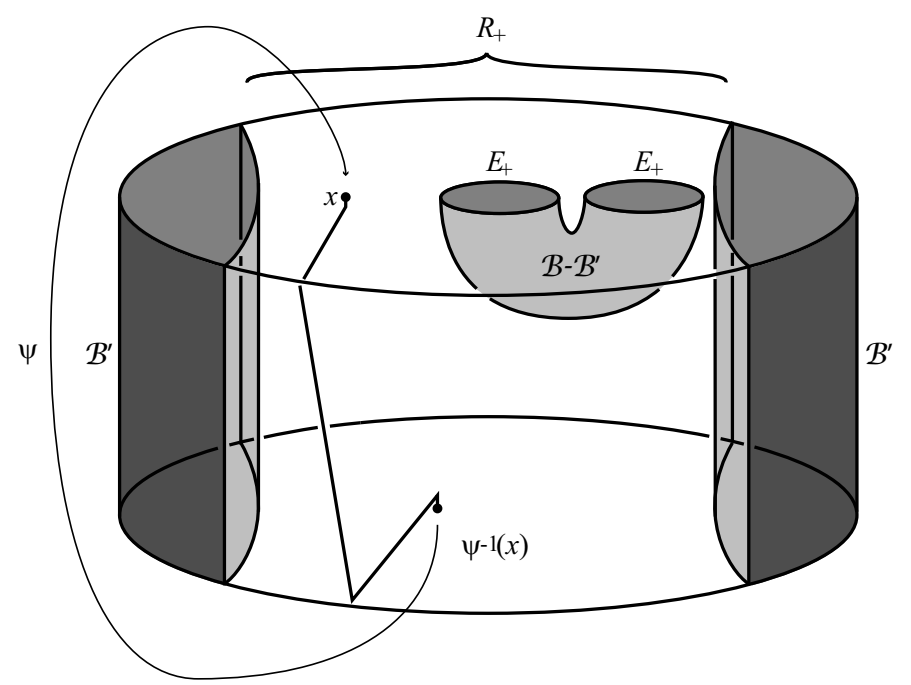

FiguRE 4

product structure on $X$. Each ball $B$ intersects $D_{+}$in a single disc and intersects $D_{-}$in a single disc. Its intersection with $S_{+}$is either empty, a disc or an annulus. In the former case, we may clearly pick a product structure on $B$ so that it agrees with product structure on $\mathcal{B}^{\prime} \cap B$. When $B \cap S_{+}$is a disc, $B$ lies in $\mathcal{B}^{\prime}$, and so it already has the desired product structure. When $B \cap S_{+}$is an annulus, one of its boundary components is disjoint from $\partial D_{+}$. This bounds a disc in $D_{+}$. Attached to this is a vertical annulus in $\mathcal{B}^{\prime}$. Its other boundary component lies in $D_{-}$, and therefore bounds a disc in $D_{-}$. The union of the discs in $D_{-}$and $D_{+}$with the vertical annulus bounds a ball in $B$ with interior disjoint from $\mathcal{B}^{\prime}$. Hence, we may extend the product structure of $\mathcal{B}^{\prime} \cap B$ over this ball, to give the required product structure on $B$. This proves the claim.

Note that the claim is not obviously true with $\mathcal{B}^{\prime}$ replaced by $\mathcal{B}$. For example, there may be components of $\mathcal{B}-\mathcal{B}^{\prime}$ which are disjoint from $D_{-}$or $D_{+}$. Alternatively, they may lie in $X$ in a knotted way. (See the bottom of Figure 3.)

This product structure on $X$ determines a homeomorphism $\phi_{0}: D_{+} \rightarrow D_{-}$, by first including $D_{+}$into $D \times[-1,1]$ and then projecting onto $D \times\{-1\}=D_{-}$. We also have a homeomorphism $\psi: D_{-} \rightarrow D_{+}$given by the gluing map.

Note that there was some flexibility in the choice of product structure on $X-$ $\mathcal{B}^{\prime}$, and we will now vary it a little. In fact, it is somewhat simpler to vary the homeomorphism $\phi_{0}$ to a new homeomorphism $\phi: D_{+} \rightarrow D_{-}$, which agrees with $\phi_{0}$ on $D_{+} \cap \mathcal{B}^{\prime}$ and which is isotopic to $\phi_{0}$ via an isotopy that is supported away from $D_{+} \cap \mathcal{B}^{\prime}$.

Claim 4. We may find such a homeomorphism $\phi$ with the following properties. Let $R_{+}$be any component of $D_{+}-\mathcal{B}^{\prime}$ that intersects $\partial D_{+}$. Let $R_{-}$be its image under $\phi$. Let $E_{+}$be the union of the discs in $R_{+}$bounded by the curves $R_{+} \cap \partial_{v}\left(\mathcal{B}-\mathcal{B}^{\prime}\right)$. (Possibly, $E_{+}$is empty.) Similarly, let $E_{-}$be the union of the discs in $R_{-}$bounded by the curves $R_{-} \cap \partial_{v}\left(\mathcal{B}-\mathcal{B}^{\prime}\right)$. Then, we may ensure that:

(1) $\phi\left(E_{+}\right)$is disjoint from $\psi^{-1}\left(E_{+}\right)$and

(2) $\phi^{-1}\left(E_{-}\right)$is disjoint from $\psi\left(E_{-}\right)$. 


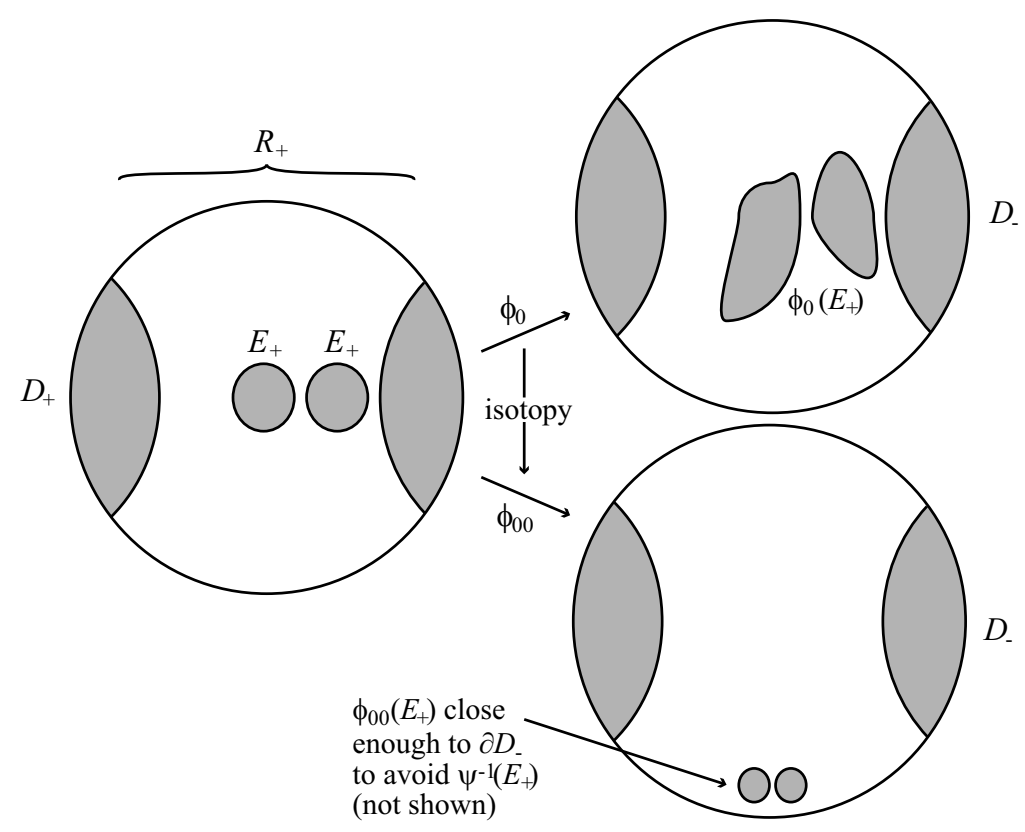

FiguRE 5

See Figure 4 for an example of $R_{+}$and $E_{+}$.

We first ensure that (1) holds. Now, $\psi^{-1}\left(E_{+}\right)$is a collection of discs in the interior of $D_{-}$. Hence, by isotoping the discs $\phi_{0}\left(E_{+}\right)$sufficiently close to $\partial D_{-}$, we may ensure that they are disjoint from $\psi^{-1}\left(E_{+}\right)$. Thus, this gives a homeomorphism $\phi_{00}: D_{+} \rightarrow D_{-}$such that $\phi_{00}\left(E_{+}\right)$is disjoint from $\psi^{-1}\left(E_{+}\right)$. (See Figure 5.)

We now ensure that (2) can also be achieved, without violating (1). All we did was isotope $\phi_{0}$ to $\phi_{00}$ so that the discs $\phi_{00}\left(E_{+}\right)$were close to $\partial D_{-}$. Hence, we may also arrange that the discs $\phi_{00}\left(E_{+}\right)$miss the discs $E_{-}$. Therefore, $\phi_{00}^{-1}\left(E_{-}\right)$is disjoint from $E_{+}$. Now, there is a 1-parameter family of homeomorphisms $D_{+} \rightarrow D_{+}$, each supported in $R_{+}$, starting at the identity, and which takes $\phi_{00}^{-1}\left(E_{-}\right)$very close to $\partial D_{+}$. In particular, we may ensure that the image of $\phi_{00}^{-1}\left(E_{-}\right)$under the final homeomorphism is disjoint from $\psi\left(E_{-}\right)$, because this is a collection of discs in the interior of $D_{+}$. Since $E_{+}$does not separate $\phi_{00}^{-1}\left(E_{-}\right)$from $\partial D_{+}$, we may assume that these homeomorphisms are the identity on $E_{+}$. Thus, pre-composing these homeomorphisms with $\phi_{00}^{-1}$, we obtain a 1-parameter family of homeomorphisms $D_{-} \rightarrow D_{+}$, starting at $\phi_{00}^{-1}$, and which equal $\phi_{00}^{-1}$ throughout when restricted to $\phi_{00}\left(E_{+}\right)$and $D_{-}-R_{-}$. Inverting, we obtain a 1 -parameter family of homeomorphisms $D_{+} \rightarrow D_{-}$, starting at $\phi_{00}$, and which equal $\phi_{00}$ throughout when restricted to $E_{+}$and $D_{+}-R_{+}$. Let $\phi: D_{+} \rightarrow D_{-}$be the final homeomorphism in this family. Since $\phi_{00}|E+=\phi| E_{+}$, condition (1) is therefore preserved. But we have ensured that $\phi^{-1}\left(E_{-}\right)$is disjoint from $\psi\left(E_{-}\right)$. This gives condition (2), which proves the claim.

Thus, we have picked a homeomorphism $\phi: D_{+} \rightarrow D_{-}$. We also have a homeomorphism $\psi: D_{-} \rightarrow D_{+}$arising from the gluing map. Consider their composition $\psi \phi: D_{+} \rightarrow D_{+}$. By the Brouwer fixed point theorem, $\psi \phi$ has a fixed point $x$ in 
$D_{+}$. After an arbitrarily small perturbation of $\phi$, we may assume that $x$ lies in the interior of a triangle or square of $D_{+}$.

The proof now divides into three cases:

1. $x \in \mathcal{B}^{\prime}$;

2. $x$ lies in a component of $D_{+}-\mathcal{B}^{\prime}$ that has non-empty intersection with $\partial D_{+} ;$

3. $x$ lies in a component of $D_{+}-\mathcal{B}^{\prime}$ that is disjoint from $\partial D_{+}$.

Case 1. $x \in \mathcal{B}^{\prime}$.

Then the endpoints of the arc $x \times[-1,1]$ patch together to form a core curve. But this leads to a contradiction in this case, because the two endpoints of $x \times[-1,1]$ lie in distinct normal discs of $D$.

Case 2. $x$ lies in a component of $D_{+}-\mathcal{B}^{\prime}$ that has non-empty intersection with $\partial D_{+}$.

Let $R_{+}$be the component of $D_{+}-\mathcal{B}^{\prime}$ containing $x$, and let $R_{-}=\phi\left(R_{+}\right)$. Define $E_{+}$and $E_{-}$as in Claim 4. An example of such a configuration is shown in Figure 4.

Then $x$ is disjoint from $\mathcal{B}$ and lies in the component of $R_{+}-\mathcal{B}$ that has nonempty intersection with $\partial D_{+}$. For otherwise, $x$ lies in $E_{+}$. By (1) in Claim 4, $\psi \phi\left(E_{+}\right)$is disjoint from $E_{+}$. In particular, $\psi \phi(x) \neq x$, which is a contradiction.

Similarly, $\phi(x)$ is disjoint from $\mathcal{B}$ and lies in the component of $R_{-}-\mathcal{B}$ that has non-empty intersection with $\partial D_{-}$. For otherwise, $\phi(x)$ lies in $E_{-}$. Hence, $x$ lies in $\phi^{-1}\left(E_{-}\right)$. Also $\psi \phi(x)$ lies in $\psi\left(E_{-}\right)$. But, by $(2)$ in Claim $4, \phi^{-1}\left(E_{-}\right)$and $\psi\left(E_{-}\right)$ are disjoint.

We will shortly construct our pre-core curve $C$. We will pick an arc in $A-\mathcal{B}$ that runs from $\partial R_{+}$to $\partial R_{-}$. We will then attach $\operatorname{arcs}$ in $D_{+}-\mathcal{B}$ and $D_{-}-\mathcal{B}$ that run to $x$ and $\phi(x)=\psi^{-1}(x)$ respectively. These three arcs patch together to form an arc with one endpoint in $D_{+}$and one in $D_{-}$. Gluing the two ends of this arc will form the pre-core curve $C$. (See Figure 4.)

We first construct a pre-core curve $C_{0}$. The curves $C$ and $C^{\prime}$ required by Theorems 1.1 and 1.2 will be minor modifications of this. We wish to arrange that $C_{0}$ sits well with respect to $T$. Now, $D_{+}, D_{-}$and $A$ clearly inherit cell structures from $T$. (For example, each 2-cell of $D_{+}$and $D_{-}$is a normal triangle or square.) Note that whenever a $k$-cell of $A$ (respectively, $D_{-} \cup D_{+}$) lies in $\mathcal{B}$, then every incident $j$-cell of $A$ (respectively, $D_{-} \cup D_{+}$), with $j<k$, also lies in $\mathcal{B}$. Thus, we may arrange that $C_{0} \cap A$ intersects only the 2-cells and 1-cells and that it is transverse to the 1-cells. We ensure that $C_{0} \cap A$ intersects the 1-cells in as few points as possible, among all such arcs in $A-\mathcal{B}$ joining $R_{+}$to $R_{-}$. Similarly, we may arrange that $C_{0} \cap\left(D_{-} \cup D_{+}\right)$intersects only the 2-cells and 1-cells of these cell structures and that it is transverse to the 1-cells. We choose these $\operatorname{arcs} C_{0} \cap\left(D_{-}-\mathcal{B}\right)$ and $C_{0} \cap\left(D_{+}-\mathcal{B}\right)$ to have the shortest possible length among all arcs joining the endpoints of $C_{0} \cap A$ to $x$ and $\phi(x)$ satisfying the above conditions. This has various consequences:

1. The intersection of $C_{0}$ with each cell of $D_{+}, D_{-}$and $A$ is connected whenever it is non-empty.

2. If $C_{0}$ intersects a 2-cell of $A$ that has non-empty intersection with $R_{+}$or $R_{-}$, then the relevant endpoint of $C_{0} \cap A$ lies in that 2-cell.

3. $C_{0}$ is disjoint from each 1-cell of $A$ that has an endpoint in $R_{-} \cup R_{+}$but does not lie entirely in $R_{-} \cup R_{+}$. 
Now, $C_{0}$ is not in the form required by either Theorem 1.1 or 1.2 , because when it lies in $\partial M$, it lies in the 2-skeleton of $T$, but when it runs through the interior of $M$, it is transverse to the 2 -skeleton. We therefore now explain how to modify $C_{0}$.

Our first aim is to create the core curve $C$. Let $C$ initially be $C_{0}$. Now shorten $C \cap D_{+}$a little at the $x$ end, so that it terminates in the interior of a 1-cell of $D_{+}$. We correspondingly lengthen $C \cap D_{-}$. We also modify $C \cap A$ near its endpoints so that it ends on 0 -cells of $A \cap\left(\partial D_{-} \cup \partial D_{+}\right)$. We now homotope $C \cap\left(D_{-} \cup D_{+}\right)$into the 1-skeleton of $D_{-} \cup D_{+}$, keeping the endpoints of these arcs fixed. This may create non-embedded arcs, but if so there is an obvious way to shorten them. Thus, we may assume that $C \cap\left(D_{-} \cup D_{+}\right)$is two embedded arcs. Hence, $C$ is a pre-core curve. Note that $C \cap\left(D_{-} \cup D_{+}\right)$need not be disjoint from $\mathcal{B}$. However, each 1-cell that it runs over is adjacent to a 2 -cell with interior disjoint from $\mathcal{B}$. Note that $C$ lies in the 2-skeleton of $T$, misses the vertices of $T$ and intersects the edges of $T$ in only finitely many points. Thus, the following claim will prove Theorem 1.1.

Claim 5. $C$ intersects the interior of each face of $T$ in at most 10 straight arcs.

The arcs of $C$ come in two types: parts lying in $A$ and parts lying in $D_{-} \cup D_{+}$. By construction, both types of arcs are straight. Thus, we only need to bound the number of such arcs in any face of $T$. Consider any such face $F$. The intersection $F \cap D$ consists of at most 3 types of normal arcs. If a normal arc type does not arise, we add one in, for the sake of streamlining the argument. By adding in such an arc very close to a vertex of $F$, we can avoid it intersecting $C$. Thus, $F-N(D)$ consists of three types of region:

1. triangular regions containing a vertex of $F$;

2. a hexagonal region containing all three arc types in its boundary;

3. rectangular regions between parallel arcs of $F \cap D$, which we call parallelity rectangles.

The parallelity rectangles lie in $\mathcal{B}$, but $C$ need not be disjoint from $\mathcal{B}$. However, the parts of $C$ that lie in $\mathcal{B}$ are in $D_{-} \cup D_{+}$and are adjacent to 2-cells of $D_{-} \cup D_{+}$ with interior disjoint from $\mathcal{B}$. Thus, if $C$ intersects a parallelity rectangle, then in one of the adjacent 3 -simplices the parallelity rectangle lies between a triangle and a square of $D$. This can happen only once in each of the adjacent 3 -simplices. Thus, $C$ intersects at most 2 parallelity rectangles, and when it intersects a parallelity rectangle, it does so in at most 2 arcs. It intersects each triangular region in at most one arc. It intersects the hexagonal region in at most 3 arcs. So, the total number of arcs of $C \cap F$ is at most 10, which proves the claim.

We now explain how to construct the core curve $C^{\prime}$ required by Theorem 1.2. We simply push $C_{0} \cap A$ a little into the interior of $M$. This can be done in such a way that $C^{\prime}$ intersects each 3 -simplex of $T$ in a collection of properly embedded arcs.

Claim 6. $C^{\prime}$ intersects each 3 -simplex of $T$ in at most 18 arcs.

Let $\Delta$ be a 3 -simplex of $T$, and let $P$ be the closure of a component of $\Delta-N(D)$. Then, the boundary of $P$ consists of triangles and squares of $D_{-} \cup D_{+}$, together with bits lying in $\partial \Delta$. The intersection $P \cap C_{0}$ is a union of the following pieces:

(1) arcs lying in $\partial \Delta$, which we call boundary arcs;

(2) arcs lying in a copy of a normal triangle or square of $D$, which we call interior arcs; 


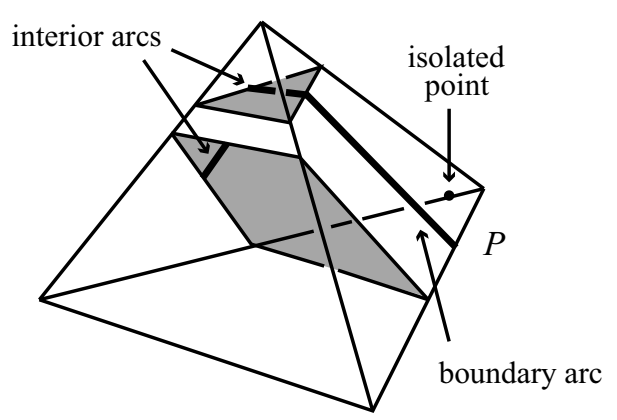

Figure 6

(3) isolated points in edges of $\partial \Delta$ that lie in $\partial M$.

It is possible for interior arcs and boundary arcs to meet at their endpoints and for boundary arcs to be joined together, but otherwise these pieces are disjoint. (See Figure 6.)

Note that isolated points of $P \cap C_{0}$ arise as follows. When $C_{0} \cap A$ runs over an edge $e$ of $T$, all the adjacent 3-simplices of $T$ pick up a point of intersection with $C_{0}$. If $\Delta$ is one of these 3 -simplices and neither of the faces of $\Delta$ containing $e$ lies in $\partial M$, this gives rise to an isolated point of $\Delta \cap C_{0}$.

Let $\Delta^{1}$ be the edges of $\Delta$, with the vertices removed.

In order to bound the number of components of $\Delta \cap C_{0}$, the first thing that we do is disregard all boundary arcs that lie in the same component of $\Delta \cap C_{0}$ as an interior arc. We do not remove these arcs from $C_{0}$; we simply suppose that they are not there, for the purposes of this counting argument. This has the possible effect of increasing the number of components of $\Delta \cap C_{0}$, but it does not decrease this number. Once this has been done, every component of $\Delta \cap C_{0}$ is either an interior arc or has non-empty intersection with $\Delta^{1}$. Thus, the number of components of $\Delta \cap C_{0}$ is at most the number of interior arcs plus the number of intersections between $C_{0}$ and $\Delta^{1}$.

Now, each edge of $\Delta$ is divided into arcs by $D$. If one of these arcs is disjoint from the vertices of $\Delta$, then it lies in $\mathcal{B}$. Thus, the only components of $\Delta^{1}-N(D)$ that can intersect $C_{0}$ are those that are incident to a vertex of $\Delta$. Call such components of $\Delta^{1}-N(D)$ vertex-incident. There are at most 12 vertex-incident arcs of $\Delta^{1}-N(D)$. By property (1) of $C_{0}$ given above, $C_{0}$ can intersect each arc of $\Delta^{1}-N(D)$ in at most one point. So there are at most 12 points of $C_{0} \cap \Delta^{1}$.

Between adjacent normally parallel triangles or squares of $\Delta \cap D$ lies $\mathcal{B}$. Hence, $C_{0}$ misses this region. So, each normal disc type of $\Delta \cap D$ can give rise to at most two interior arcs. There are at most 5 normal disc types in $\Delta$, and so there are at most 10 interior arcs of $C_{0} \cap \Delta$.

Thus, the total number of components of $C_{0} \cap \Delta$ is at most $12+10=22$. But we can reduce this bound down to 18 as follows.

Each interior arc of $\Delta \cap C_{0}$ lies in a normal disc $E$ of $D_{-} \cup D_{+}$. Then $E$ lies in $R_{-} \cup R_{+}$, and so the components of $\Delta^{1}-N(D)$ incident to $E$ are disjoint from $C_{0}$, by condition (3) above. When such a component of $\Delta^{1}-N(D)$ is also vertex-incident, this reduces the number of possible points of $C_{0} \cap \Delta^{1}$ below 12. Now, 6 discs of $\left(D_{-} \cup D_{+}\right) \cap \Delta$ that are not contained in $\mathcal{B}$ may be disjoint from vertex-incident 
arcs of $\Delta^{1}-N(D)$. But if there are any more than 6 interior arcs of $\Delta \cap C_{0}$, then each one reduces the number of possible points of $\Delta^{1} \cap C_{0}$ by at least 3 . So, we deduce that the total number of components of $C_{0} \cap \Delta$ is at most $12+6=18$. Each arc of $\Delta \cap C^{\prime}$ comes from a component of $\Delta \cap C_{0}$. This proves the claim.

Claim 7. $C^{\prime} \cap \Delta$ is parallel to a collection of $\operatorname{arcs} \alpha$ in $\partial \Delta$, with the property that the intersection between each component of $\alpha$ and each face of $\Delta$ is at most one straight arc.

We have already arranged that $C^{\prime}$ intersects each 3 -simplex in one of only finitely many possible configurations. So, one could simply perform a case-by-case check to prove this claim. However, there are many cases, and so we present a uniform argument.

Let $\Delta$ be a 3-simplex of $T$, and let $P$ be the closure of a component of $\Delta-N(D)$. We first show that $C^{\prime} \cap P$ is parallel in $P$ to a collection of $\operatorname{arcs} \alpha$ in $\partial \Delta \cap P$. We will then show that $\alpha$ can be chosen so that each component intersects each face of $\Delta$ in at most one straight arc. This will prove the claim.

Now, the boundary arcs and isolated points of $C_{0} \cap P$ already lie in $\partial \Delta$. These will form part of $\alpha$. Thus, we need only to worry about the parts of $C_{0} \cap P$ lying in interior arcs. There is at most one interior arc lying in each normal triangle or square of $D_{-} \cup D_{+}$, and this can be slid, keeping its endpoints fixed, into the boundary of the triangle or square. There are two possible directions in which this slide can be performed, and we choose a direction which minimises the number of intersections with $\Delta^{1}$. This creates our choice of $\operatorname{arcs} \alpha$.

How could a component of $\alpha$ intersect a face of $\Delta$ in more than one component? Each component of $C_{0} \cap \Delta$ intersects each face of $\Delta$ in at most one component. Thus, the only possible problem that could arise is when the process of sliding creates a new component of intersection with a face. This only happens when the interior arc of $C_{0} \cap \Delta$ lies in a normal square $E$ and the interior arc joins opposite edges of the square. Then one of the sides of the square $(\beta$, say) becomes a new component of intersection between $\alpha$ and a face of $\Delta$. Now, each component of $\Delta-D$ that does not lie wholly in $\mathcal{B}$ contains at most one normal square of $D_{-} \cup D_{+}$. So, the only way that a problem could arise is when the 2-cell of $A$ containing $\beta$ has non-empty intersection with $C_{0}$. But in this case, condition (2) above implies that the relevant endpoint of $C_{0} \cap A$ lies in that 2-cell. This can happen in only that 2-cell. So, if we slide $C_{0} \cap E$ the other way across $E$, then this problem is avoided. Finally, note that $\alpha$ intersects each face of $P$ in at most one arc. We may therefore isotope $\alpha$ so that it is straight in each face. This proves the claim.

Thus, we have proved Theorems 1.1 and 1.2 in this case.

Case 3. $x$ lies in a component of $D_{+}-\mathcal{B}^{\prime}$ that is disjoint from $\partial D_{+}$.

An example of such a configuration is shown in Figure 7.

Let $R_{+}$be the closure of the component of $D_{+}-\mathcal{B}^{\prime}$ containing $x$. This is a disc disjoint from $\partial D_{+}$. Since $\psi \phi(x)=x, \psi \phi\left(R_{+}\right)$has non-empty intersection with $R_{+}$. We consider two cases.

Case 3A. $\psi \phi\left(\partial R_{+}\right) \cap \partial R_{+} \neq \emptyset$.

Thus, $\psi \phi\left(\partial R_{+}\right) \cap \partial R_{+}$contains some point $y$. If we were to pick an arc in the annulus $\partial R_{+} \times[-1,1]$ running from $y$ to $\psi^{-1}(y)$, then this would patch up to form a core curve for $M$. In fact, we will feel free to vary this arc by an ambient isotopy, 


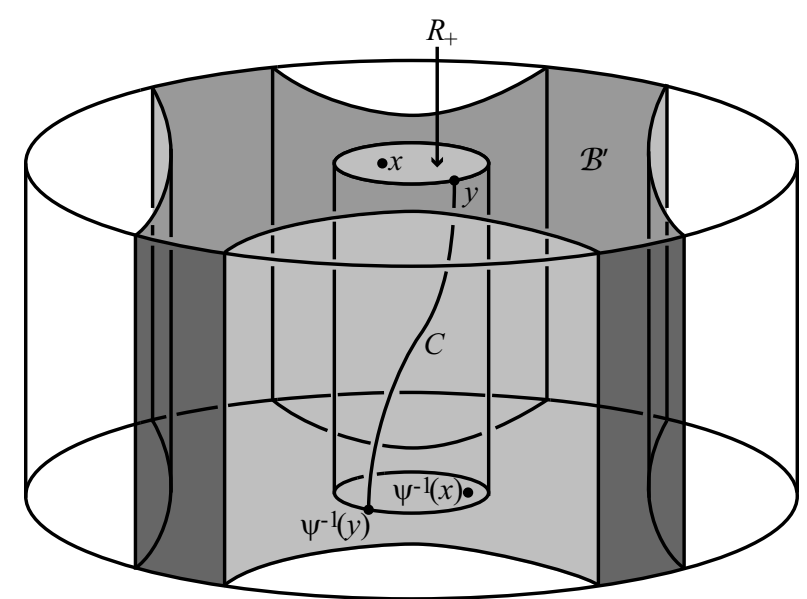

FiguRE 7

keeping its endpoints fixed. The arc first runs from $y$ to $\phi(y)$ vertically across $\partial_{v} \mathcal{B}$. It then runs from $\phi(y)$ to $\psi^{-1}(y)$ along the 1-cells of $D_{-}$. Each such 1-cell is adjacent to a 2-cell with interior disjoint from $\mathcal{B}$. So, as in Case 2, the resulting core curve $C$ lies in the 2-skeleton of $T$ and intersects the interior of each face of $T$ in at most 10 straight arcs. One may also perturb $C$ to create a core curve $C^{\prime}$ that is transverse to the 2-skeleton of $T$. It then intersects each tetrahedron only in interior arcs, as described in Case 2. So, $C^{\prime}$ intersects each tetrahedron in at most 10 arcs, and these satisfy the conclusions of Theorem 1.2. Thus, the theorems are proved in this case.

Case 3B. $\psi \phi\left(\partial R_{+}\right) \cap \partial R_{+}=\emptyset$.

Now, $\psi \phi\left(R_{+}\right)$has non-empty intersection with $R_{+}$, and yet their boundaries are disjoint, and so they must be nested. Say that $\psi \phi\left(R_{+}\right)$lies in $R_{+}$.

Recall that $\widehat{\mathcal{H}}$ is the handle structure on $M$ that is obtained from $T$ by thickening each $i$-simplex to form an $i$-handle. Note that $D_{+}$inherits a handle structure from $\widehat{\mathcal{H}}$. Both $R_{+}$and $\psi \phi\left(R_{+}\right)$are a union of handles in this handle structure. However, $R_{+}$and $\psi \phi\left(R_{+}\right)$do not inherit handle structures. This is because it need not be true that whenever an $i$-handle lies in $R_{+}$(or $\psi \phi\left(R_{+}\right)$), then so does every $j$-handle to which it is attached, with $j<i$. In fact, $\operatorname{cl}\left(D_{+}-R_{+}\right)$and $\operatorname{cl}\left(D_{+}-\psi \phi\left(R_{+}\right)\right)$ inherit handle structures. This is because the handles of $D_{+}$that intersect $R_{+}$but do not lie in it are actually part of $\mathcal{B}$. Also, whenever an $i$-handle lies in $\mathcal{B}$, then so is every $j$-handle to which it is attached, with $j<i$.

We now consider the weight of $R_{+}$and $\psi \phi\left(R_{+}\right)$. Recall that this is just the number of intersections with the 1-skeleton of the triangulation. Equivalently, it is the number of 0-handles of $R_{+}$(or $\psi \phi\left(R_{+}\right)$).

Claim 8. $\psi \phi\left(R_{+}\right)$has strictly smaller weight than $R_{+}$.

Now, $\psi \phi\left(R_{+}\right)$is a subset of $R_{+}$consisting of a union of handles. Thus, if the weight of $\psi \phi\left(R_{+}\right)$is not less than that of $R_{+}$, then only 1-handles and 2-handles are removed when constructing $\psi \phi\left(R_{+}\right)$from $R_{+}$. But $\psi \phi\left(\partial R_{+}\right)$is disjoint from $\partial R_{+}$, and so every handle of $R_{+}$that contains $\partial R_{+}$is removed. Also, if an $i$-handle 
is removed, then so is every $j$-handle that it is attached to, whenever $j<i$. Now, if there is no 0 -handle of $R_{+}$that is incident to a 1-handle or 2-handle which contains an arc of $\partial R_{+}$, then $R_{+}$consists only of 1-handles and 2-handles. But in this case, every handle of $R_{+}$has non-empty intersection with $\partial R_{+}$, and so all of $R_{+}$ is removed when creating $\psi \phi\left(R_{+}\right)$. In other words, $\psi \phi\left(R_{+}\right)$is empty, which is a contradiction. This proves the claim.

So, consider the disc $D^{\prime}$ obtained from $D$ by removing the interior of $R_{+}$and then attaching $\left(\partial R_{+} \times[-1,1]\right) \cup \psi \phi\left(R_{+}\right)$. Since $\partial R_{+} \times[-1,1]$ is vertical in the parallelity bundle, it has zero weight. Thus, $D^{\prime}$ has the same boundary as $D$ but has smaller weight. This contradicts our assumption that $D$ has minimal complexity.

\section{Partially ideal triangulations and AFFine handle structures}

In this section, we generalise Theorems 1.1 and 1.2 from triangulations to two other types of representation of a 3-manifold: partially ideal triangulations and affine handle structures.

A partially ideal triangulation of a 3-manifold $M$ is an expression of $M-\partial M$ as a collection of 3-simplices with their faces identified in pairs, and then with some of their vertices removed. We will assume, without loss of generality, that all of the gluing maps are affine. Hence, as in previous sections, it makes sense to speak of a straight line in a face of the triangulation.

One can remove a small open product neighbourhood of each end of $M-\partial M$, thereby truncating the ideal vertices of each tetrahedron. This can be made into a convex polyhedron by starting with a regular Euclidean tetrahedron and truncating some its vertices. We call this a truncated partially ideal triangulation.

We will prove the following version of Theorem 1.2 in this context.

Theorem 4.1. Let $T$ be a truncated partially ideal triangulation of the solid torus $M$. Then there is a core curve $C$ of $M$ that intersects each truncated tetrahedron $\Delta$ of $T$ in a collection of at most 48 properly embedded arcs, with endpoints in the interiors of the faces of $\Delta$. These arcs $C \cap \Delta$ are simultaneously parallel to a union of arcs in $\partial \Delta$, each of which intersects each face in at most 6 straight lines.

We will also deal with handle structures. Whenever we refer to a handle structure on a 3-manifold, we insist that each handle is attached to handles of strictly lower index.

In order to be able to refer to straight curves in a 0-handle or 1-handle, we introduce the following new concept. An affine handle structure on a 3-manifold $M$ is a handle structure where each 0 -handle and 1-handle is identified with a compact polyhedron in $\mathbb{R}^{3}$, so that

1. each face of each polyhedron is convex (but the polyhedron identified with a 0 -handle need not be convex);

2. whenever a 0-handle and 1-handle intersect, each component of intersection is identified with a convex polygon in $\mathbb{R}^{2}$ in such a way that the inclusion of this intersection into each handle is an affine map onto a face of the relevant polyhedron;

3. for each 0-handle $H_{0}$, each component of intersection with a 2-handle, 3handle or $\partial M$ is a union of faces of the polyhedron associated with $H_{0}$;

4. the polyhedral structure on each 1-handle is the product of a convex 2dimensional polygon and an interval. 
Here, we have the following version of Theorem 1.2.

Theorem 4.2. Let $\mathcal{H}$ be an affine handle structure of the solid torus $M$. Suppose that each 0 -handle of $\mathcal{H}$ has at most 4 components of intersection with the 1 -handles and that each 1-handle has at most 3 components of intersection with the 2-handles. Then $M$ has a core curve that intersects only the 0-handles and 1-handles, that respects the product structure on the 1-handles, that intersects each 1-handle in at most 24 straight arcs, and that intersects each 0-handle in at most 48 arcs. Moreover, the arcs in each 0-handle are simultaneously parallel to a collection of arcs $\alpha$ in the boundary of the corresponding polyhedron, and each component of $\alpha$ intersects each face of the polyhedron in at most 6 straight arcs.

The definition of an affine handle structure may not seem to be particularly natural. For example, it is not clear why one only identifies the 0-handles and 1handles with polyhedra. However, to impose a polyhedral structure on the handles with higher index would be unduly restrictive and also unnecesssary. Similarly, it is necessary for us to insist that the faces of each polyhedron are convex, but not that the polyhedra themselves are convex.

In Theorem 4.2, the restrictions on the number of components of intersection between various types of handle may also seem strange. But the conditions are natural. For example, they are satisfied by the handle structure that is dual to a partially ideal triangulation. In fact, Theorem 4.2 is a generalisation of Theorem 4.1. For, suppose that we are given a truncated partially ideal triangulation of a 3-manifold. We then declare that each truncated tetrahedron is a 0-handle. Each face of the ideal triangulation is made into a 1-handle. Each edge becomes a 2handle, and each non-ideal vertex becomes a 3 -handle. Theorem 4.2 then provides a core curve that intersects this handle structure nicely. Translating this back to the initial ideal triangulation, we obtain the conclusion of Theorem 4.1.

We therefore focus on the proof of Theorem 4.2. Let $\mathcal{H}$ be the given handle structure, and for $i=0,1,2,3$, let $\mathcal{H}^{i}$ be the union of the $i$-handles.

We would like to be able to assume that $\mathcal{H}^{0} \cap \partial M$ is a collection of discs. However, this need not be the case. But, suppose that, for some 0-handle $H_{0}, H_{0} \cap \partial M$ has a non-disc component. Pick a simple closed curve that is essential in $H_{0} \cap \partial M$. We may find such a curve that intersects each convex face of $H_{0} \cap \partial M$ in at most one straight arc. This curve bounds a properly embedded disc $E$ in $H_{0}$. There are two cases to consider: where $E$ is boundary parallel in $M$ and where $E$ is a meridian disc for $M$. If $E$ is boundary parallel, then the parallelity region is a 3-ball. Remove from $\mathcal{H}$ all the handles that lie in this ball, except $H_{0}$. It is clear that if the theorem holds for this new handle structure, then it also holds for $\mathcal{H}$. Thus, we may assume that this case does not arise. Suppose now that $E$ is a meridian disc. Then the result of cutting $\partial M$ along $\partial E$ is an annulus. If we pick any properly embedded arc in this annulus joining its two boundary components, then this can be closed up to form a pre-core curve $C$. We may clearly arrange it so that $C$ intersects only the 0 -handles and 1-handles of $\mathcal{H}$ and that it respects the product structure on the 1-handles. We may also ensure that it runs over each component of $\mathcal{H}^{1} \cap \partial M$ at most once. Thus, it runs over each 1-handle of $\mathcal{H}$ at most three times. The points where it enters and leaves any 0 -handle lie in $\mathcal{H}^{1}$, and so it intersects each 0 -handle in at most 6 arcs. We may ensure that each such arc intersects each face of $\partial \mathcal{H}^{0}$ in at most one straight arc. Now push $C$ a little into the interior of $M$ to create the core curve required by Theorem 4.2. 
We may therefore assume that $\mathcal{H}^{0} \cap \partial M$ is a collection of discs. This forces each 2-handle to run over at least one 1-handle. Therefore, $\mathcal{H}^{0} \cap\left(\mathcal{H}^{1} \cup \mathcal{H}^{2}\right)$ forms a thickened graph, where $\mathcal{H}^{0} \cap \mathcal{H}^{1}$ is the thickened vertices and $\mathcal{H}^{0} \cap \mathcal{H}^{2}$ is the thickened edges.

In the proof of Theorems 1.1 and 1.2, a handle structure was constructed from the triangulation of $M$, and this was used to define the parallelity bundle. We want to do something similar here. The result will be a handle structure $\widehat{\mathcal{H}}$ which is, in some sense, dual to the given handle structure $\mathcal{H}$. Handles of $\widehat{\mathcal{H}}$ will arise in two possible ways. Each $i$-handle of $\mathcal{H}$ will give rise to a $(3-i)$-handle of $\widehat{\mathcal{H}}$. But there is a second type of handle of $\widehat{\mathcal{H}}$. The handle structure $\mathcal{H}$ induces a handle structure for $\partial M$, and each $i$-handle of $\partial M$ gives rise to a $(2-i)$-handle of $\widehat{\mathcal{H}}$. So, a handle of $\mathcal{H}$ that meets $\partial M$ may give rise to several handles of $\widehat{\mathcal{H}}$. For instance, consider a 2-handle $D^{2} \times D^{1}$ of $\mathcal{H}$ such that $D^{2} \times \partial D^{1} \subset \partial M$. In $\widehat{\mathcal{H}}$, this becomes a 1 -handle connecting two 0-handles.

One may also form a cell structure for $M$ by collapsing each $i$-handle of $\widehat{\mathcal{H}}$ to an $i$-cell.

The first step in the proof of Theorems 1.1 and 1.2 was to find a meridian disc $D$ in normal form. The complexity of $D$ was defined to be an ordered pair of integers: the length of $\partial D$ and the weight of $D$. Complexities were ordered lexicographically, and $D$ was chosen to have the smallest possible complexity. We perform a similar process in this case.

We start by making $D$ standard in the handle structure $\mathcal{H}$. This means that it intersects each handle in a collection of properly embedded discs, misses the 3handles, and respects the product structure on each 2-handle and 1-handle. We define the boundary weight of $D$ to be the number of intersections between $D$ and $\partial M \cap \mathcal{H}^{0}$. We define the interior weight to be the number of intersections between $D$ and $\mathcal{H}^{2}$. We define the compexity of $D$ to be the ordered pair, boundary weight and then interior weight, and choose $D$ so that it has minimal complexity.

The resulting disc $D$ need not be normal, in the usual sense of the word (for example, as in [3] or [5]). This is because there may be arcs of intersection between $D$ and a component of $\mathcal{H}^{0} \cap \mathcal{H}^{1}$ which run from a component of $\mathcal{H}^{0} \cap \partial M$ to an adjacent component of $\mathcal{H}^{0} \cap \mathcal{H}^{2}$. But $D$ satisfies the other conditions of normality:

(1) $D \cap \mathcal{H}^{0} \cap \mathcal{H}^{1}$ consists of arcs;

(2) $D \cap \mathcal{H}^{0} \cap \mathcal{H}^{2}$ consists of arcs respecting the product structure in $\mathcal{H}^{0} \cap \mathcal{H}^{2}$;

(3) $D \cap \mathcal{H}^{0} \cap \partial M$ consists of arcs;

(4) no arc of intersection between $D$ and $\mathcal{H}^{0} \cap \mathcal{H}^{1}$ has endpoints in the same component of $\mathcal{H}^{0} \cap \mathcal{H}^{1} \cap \mathcal{H}^{2}$ or in the same component of $\mathcal{H}^{0} \cap \mathcal{H}^{1} \cap \partial M$;

(5) no arc of intersection between $D$ and $\mathcal{H}^{0} \cap \partial M$ has endpoints in the same component of $\mathcal{H}^{0} \cap \mathcal{H}^{1} \cap \partial M$;

(6) each component of $D \cap \partial \mathcal{H}^{0}$ intersects each component of $\mathcal{H}^{0} \cap \mathcal{H}^{2}$ in at most one arc;

(7) each component of $D \cap \partial \mathcal{H}^{0}$ intersects each component of $\mathcal{H}^{0} \cap \partial M$ in at most one arc.

For any handle $H$ of $\mathcal{H}$, we say that two discs of $H \cap D$ are of the same type if there is an ambient isotopy, preserving all the handles of $\mathcal{H}$, that takes one disc to the other. The above conditions imply that $D$ intersects each handle in only finitely many disc types. By condition (6), each disc $E$ of $D \cap \mathcal{H}^{0}$ intersects each 
component of $\mathcal{H}^{0} \cap \mathcal{H}^{2}$ in at most one arc. By condition (7), $E$ intersects each component of $\mathcal{H}^{0} \cap \partial M$ in at most one arc. By a combination of conditions (4), (6) and (7) and the assumption that each 1-handle has at most 3 components of intersection with the 2-handles, $E$ intersects each component of $\mathcal{H}^{0} \cap \mathcal{H}^{1}$ in at most 3 arcs.

We now make $D$ sit nicely with respect to $\widehat{\mathcal{H}}$. Each component of intersection between $D$ and a handle of $\mathcal{H}$ or $\partial M$ gives rise to a disc component of intersection between $D$ and a handle of $\widehat{\mathcal{H}}$. We define the type of such a disc just as in the case of $\mathcal{H}$. Between adjacent discs of the same type, there is a product region, and these patch together to form the parallelity bundle $\mathcal{B}$.

Now, each 0 -handle $H_{0}$ of $\mathcal{H}$ has been identified with a polyhedron, and each component of $H_{0} \cap \mathcal{H}^{1}, H_{0} \cap \mathcal{H}^{2}$ and $H_{0} \cap \partial M$ is a union of convex faces. We may clearly arrange that $D$ intersects each such convex face in a collection of straight arcs. Moreover, we may ensure that each component of $D \cap H_{0} \cap \mathcal{H}^{1}, D \cap H_{0} \cap \mathcal{H}^{2}$ and $D \cap H_{0} \cap \partial M$ intersects each convex face in at most one such arc.

The rest of the argument proceeds as in the case of triangulations, apart from the exact specification of the core curve $C$ with respect to $\mathcal{H}$. The symbols $D_{-}, D_{+}, A$, $X$ and $\mathcal{B}^{\prime}$ denote exactly what they did before. A homeomorphism $\phi: D_{+} \rightarrow D_{-}$is picked, and $\psi: D_{-} \rightarrow D_{+}$is the gluing map. The composition $\psi \phi: D_{+} \rightarrow D_{+}$has a fixed point $x$. The proof then divides into cases according to the location of $x$. As before, there are two cases when a copy of $C$ is constructed (Cases 2 and $3 \mathrm{~A}$ ). We will focus on Case 2, because this is more complex. Recall that this is when $x$ lies in a component of $D_{+}-\mathcal{B}^{\prime}$ that has non-empty intersection with $\partial D_{+}$. Let $R_{+}$be the closure of the component of $D_{+}-\mathcal{B}^{\prime}$ containing $x$, and let $R_{-}=\phi\left(R_{+}\right)$. Recall that in this case, we picked a curve $C_{0}$ in $M$, avoiding $\mathcal{B}$, as follows. The arc $C_{0} \cap A$ ran between $R_{-}$and $R_{+}$. It was disjoint from the 0 -cells of $A$ and was transverse to the 1-cells. It was chosen to have the fewest number of intersections with the 1-cells. Then $C_{0} \cap\left(D_{+}-\mathcal{B}\right)$ and $C_{0} \cap\left(D_{-}-\mathcal{B}\right)$ were arcs running from the endpoints of $C_{0} \cap A$ to $x$ and $\phi(x)$. These were also chosen to have the smallest length among all curves in $D_{ \pm}-\mathcal{B}$ joining these specified endpoints, in the sense of having the fewest number of points of intersection with the 1-cells.

In our situation, we do the same. Once again, $A, D_{-}$and $D_{+}$inherit cell structures. For example, each component of intersection between $D$ and $\mathcal{H}^{0}$ gives a component of intersection between $D$ and a 3 -handle of $\widehat{\mathcal{H}}$, and this becomes a 2-cell of $D_{-}$and $D_{+}$. The arc $C_{0} \cap A$ is chosen to have the smallest length among all curves in $A-\mathcal{B}$ joining $R_{-}$to $R_{+}$. We then pick the shortest $\operatorname{arcs}$ in $\left(D_{-}-\mathcal{B}\right)$ and $\left(D_{+}-\mathcal{B}\right)$ joining the endpoints of $C_{0} \cap A$ to $x$ and $\phi(x)$. In the proof of Theorem 1.2 , we needed to push $C_{0}$ a little into the interior of $M$ to form a core curve. We do the same here and let $C$ be the resulting core curve.

Let $H_{0}$ be a 0 -handle of $\mathcal{H}$. We wish to find an upper bound on the number of arcs of $C_{0} \cap H_{0}$. To do this, we note that the only places where $C_{0}$ can enter $H_{0}$ are in the discs $H_{0} \cap \mathcal{H}^{1}$. So, we will bound the number of points of $C_{0} \cap H_{0} \cap \mathcal{H}^{1}$. These points come in two types: those that lie on the boundary of the discs $H_{0} \cap \mathcal{H}^{1}$ and those that lie in the interior of these discs. The points that lie on the boundary of these discs lie in $\partial M$. So consider an arc component of $H_{0} \cap \mathcal{H}^{1} \cap \partial M$. This is divided up by $D$, and between adjacent points of $D$ there lies $\mathcal{B}$, which $C_{0}$ avoids. So, $C_{0}$ can intersect each arc of $H_{0} \cap \mathcal{H}^{1} \cap \partial M$ in at most 2 points. There are at most 3 such arcs in each component of $H_{0} \cap \mathcal{H}^{1}$, and so this gives at most 6 
endpoints of $C_{0} \cap H_{0}$. The second type of endpoint of $C_{0} \cap H_{0}$ lies in the interior of the discs of $H_{0} \cap \mathcal{H}^{1}$. These lie on an arc of $D \cap H_{0} \cap \mathcal{H}^{1}$. Between two such arcs of the same type, there again lies the parallelity bundle $\mathcal{B}$. So, each arc type of $D \cap H_{0} \cap \mathcal{H}^{1}$ gives at most two endpoints of $C_{0} \cap H_{0}$. There are at most 9 arc types in each component of $H_{0} \cap \mathcal{H}^{1}$. So, this gives at most 18 endpoints of $C_{0} \cap H_{0}$. So, in total, we have at most $6+18=24$ endpoints of $C_{0} \cap H_{0}$ in each component of $H_{0} \cap \mathcal{H}^{1}$, and hence at most $48 \operatorname{arcs}$ of $C_{0} \cap H_{0}$.

We now need to justify why $C_{0} \cap \mathcal{H}^{0}$ is parallel to a collection of arcs in $\partial \mathcal{H}^{0}$ as described in Theorem 4.2. Some of $C_{0}$ already lies in $\partial \mathcal{H}^{0}$, but we slide the parts lying in $D \cap \mathcal{H}^{0}$ into $\partial\left(D \cap \mathcal{H}^{0}\right)$. The result is a collection of $\operatorname{arcs} \alpha$ in $\partial \mathcal{H}^{0}$ to which $C_{0} \cap \mathcal{H}^{0}$ is parallel.

We must show that $\alpha$ can be isotoped, keeping $\partial \alpha$ fixed, so that it intersects each face of each polyhedron of $\mathcal{H}^{0}$ in a collection of straight arcs and that each component of $\alpha$ intersects each such face in at most 6 arcs. Now, $\partial \mathcal{H}^{0}$ is a union of convex faces, and these have been divided up by $\partial D$ into convex polygons. Thus, we may isotope $\alpha$ so that it intersects each face in straight arcs. Now, $C_{0}$ is a concatenation of interior arcs, which run over a component of $D \cap \mathcal{H}^{0}$, and boundary arcs, which run over a component of $\partial M \cap \mathcal{H}^{0}$. An interior arc is joined to a boundary arc exactly where $C_{0} \cap A$ is joined to $C_{0} \cap\left(D_{-} \cup D_{+}\right)$, and there are just two of these points. So, in each component of $C_{0} \cap \mathcal{H}^{0}$, at most one boundary arc is used, and at most two interior arcs. Now, each interior arc has been slid into the boundary of a component of $D \cap \mathcal{H}^{0}$. Each component of $D \cap \mathcal{H}^{0}$ intersects each face of $\partial \mathcal{H}^{0}$ at most 3 times. In fact, if the face lies in $\partial M$, then the component of $D \cap \mathcal{H}^{0}$ runs over it at most once. Each boundary arc lies in a single component of $\partial M \cap \mathcal{H}^{0}$, and so runs over each face at most once. Thus, each component of $\alpha$ runs over each face of $\partial \mathcal{H}^{0}$ at most 6 times, as required.

\section{RIEMANNIAN METRICS ON SOLID TORI}

In this section, we will prove that any Riemannian metric on a solid torus $M$ with bounded sectional curvature and a lower bound on injectivity radius has a core curve with length that is linearly bounded by the volume of $M$.

Theorem 1.3. For each $K, I>0$, there is a constant $c(K, I)$ with the following property. If $M$ is a solid torus with a Riemannian metric having volume at most $V$, injectivity radius at least $I$ and all sectional curvatures in the interval $(-K, K)$, then $M$ contains a core curve with length at most $c(K, I) V$.

This is proved by approximating (in a certain sense) the Riemannian metric by a triangulation, as follows.

Proposition 5.1. For each $K, I>0$, there is a constant $c^{\prime}(K, I)$ with the following property. If $M$ is a compact 3-manifold with a Riemannian metric having injectivity radius at least $I$ and all sectional curvatures in the interval $(-K, K)$, then $M$ has a triangulation $T$ such that

(1) there is a $c^{\prime}(K, I)$-Lipshitz homeomorphism $M_{P L} \rightarrow M$, where $M_{P L}$ is the path metric on $M$ obtained by realising each tetrahedron of $T$ as a standard Euclidean simplex with side length 1 and

(2) the number of tetrahedra in $T$ is at most $c^{\prime}(K, I) V$, where $V$ is the volume of $M$. 

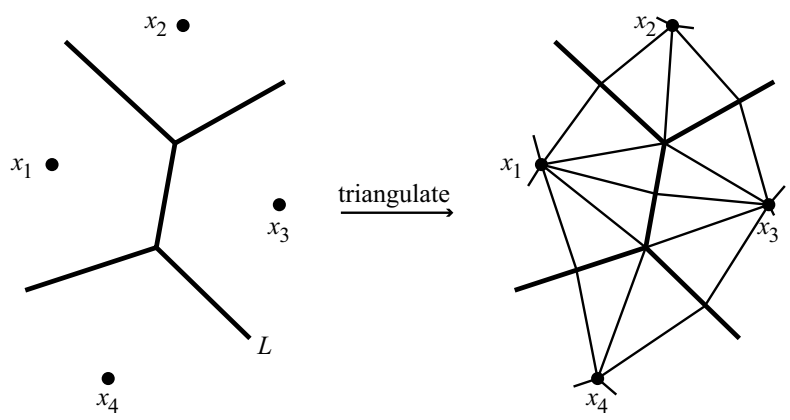

Figure 8

Proof. For each point $x$ in $M$, let $\exp _{x}: T_{x} M \rightarrow M$ be the exponential map. If we set $\epsilon>0$ to be small enough (as a function of $K$ and $I$ ), then the restriction of $\exp _{x}$ to the ball of radius $\epsilon$ about 0 is injective for all $x \in M$. Furthermore, if $\epsilon$ is sufficiently small, then $(1 / \epsilon) B_{\epsilon}(x)$ is nearly isometric to the unit ball in $\mathbb{R}^{3}$, where $B_{\epsilon}(x)$ is the ball of radius $\epsilon$ about $x$, and $(1 / \epsilon) B_{\epsilon}(x)$ is obtained from $B_{\epsilon}(x)$ by rescaling by the factor $(1 / \epsilon)$. Thus, if $x$ and $x^{\prime}$ are within distance $\epsilon$ of each other, then the set of points in $B_{\epsilon}(x)$ that are equidistant from $x$ and $x^{\prime}$ is a smooth surface, and when it is rescaled by the factor $(1 / \epsilon)$, it is nearly isometric to a subset of the Euclidean plane.

Pick a maximal set of points $\left\{x_{1}, \ldots, x_{n}\right\}$ in $M$, such that no two of these points are within $\epsilon / 2$ of each other. Then, by maximality, every point of $M$ lies at a distance of at most $\epsilon / 2$ from at least one $x_{i}$.

Now consider the cut locus $L$ of these points. More precisely, for each point $x$ in $M$, define $N(x)$ to be the number of $\left\{x_{1}, \ldots, x_{n}\right\}$ that are closest to $x$. Then $L$ is the set of points $x$ where $N(x) \geq 2$. By a small perturbation of the points $\left\{x_{1}, \ldots, x_{n}\right\}$, we may assume that $L$ is a cell complex, where the set of points $x$ with $N(x)=k$ is the open $(4-k)$-cells. A schematic picture of $L$, reduced by one dimension, is shown on the left in Figure 8.

Subdivide each 1-cell by adding in its midpoint. Each 2-cell, rescaled by $(1 / \epsilon)$, is nearly isometric to a Euclidean polygon. In particular, if $\epsilon$ is sufficiently small, it is star-shaped about some point in the interior. Subdivide the 2-cell by adding a vertex at this point and then coning off the boundary. Now subdivide each 3-cell by coning off from the point in $\left\{x_{1}, \ldots, x_{n}\right\}$ that it contains. The result is the triangulation $T$.

We now wish to bound the number of tetrahedra in $T$. Each tetrahedron has a vertex at one of $\left\{x_{1}, \ldots, x_{n}\right\}$. So our first step is to find an upper bound for $n$. The points $x_{1}, \ldots, x_{n}$ are more than $\epsilon / 2$ apart, and so the balls of radius $\epsilon / 4$ about these points are all disjoint. There is a lower bound $c_{1}(K, I)$ on the volume of each such ball. Hence, $n \leq V / c_{1}(K, I)$, where $V$ is the volume of $M$.

So, our next task is to bound the number of tetrahedra incident to some $x_{i}$. Each tetrahedron has a vertex in the interior of a 2-cell. This 2-cell is equidistant between $x_{i}$ and some $x_{j}$. These are the closest points in $\left\{x_{1}, \ldots, x_{n}\right\}$ to that vertex. But every point of $M$ is at most $\epsilon / 2$ from some point of $\left\{x_{1}, \ldots, x_{n}\right\}$. Hence, $x_{j}$ is at most $\epsilon$ from $x_{i}$. By our assumption on the $\epsilon$, the points $x_{i}$ and $x_{j}$ determine 
just one 2-cell. The number of such $x_{j}$ is at most

$$
\text { Volume }\left(B_{5 \epsilon / 4}\left(x_{i}\right)\right) / \min \left\{\operatorname{Volume}\left(B_{\epsilon / 4}\left(x_{j}\right)\right): 1 \leq j \leq n\right\},
$$

because the $\epsilon / 4$ balls about these $x_{j}$ are all disjoint and they fit into the $5 \epsilon / 4$ ball about $x_{i}$. Hence, the number of such $x_{j}$ is at most some constant $c_{2}(K, I)$.

The remaining two vertices of any tetrahedron that is incident to $x_{i}$ lie in the boundary of the 2-cell, at either a corner or the midpoint of an edge. Again, the number of such vertices is at most some constant $c_{3}(K, I)$. Hence, putting this all together, we deduce that the number of tetrahedra of $T$ is at most $c^{\prime}(K, I) V$, for some constant $c^{\prime}(K, I)$.

We now construct the Lipschitz homeomorphism $M_{P L} \rightarrow M$. Each simplex of $M_{P L}$ is already realised as a subset of $M$. So, we only need to specify how the Euclidean simplex in $M_{P L}$ is mapped into that subset. We start by sending the vertices of $M_{P L}$ to the corresponding points in $M$. Then we consider the edges of the triangulation that lie in 1-cells of $L$. We map these to $M$ so that they have constant speed. Then we consider faces of the triangulation which lie in 2-cells of $L$. These have one vertex in the interior of the 2-cell and two in the 1-skeleton. We view the face as a cone on the former vertex and map this into $M$ in a way that respects the cone structure. In other words, each point of the face lies on a unique geodesic in the Euclidean metric on the face that runs from the coning vertex to the opposite edge. We send this geodesic to the corresponding curve in the 2-cell of $L$ that is a geodesic in the path metric on $L$. We then extend the map over the remainder of each tetrahedron of $M_{P L}$ by viewing it as a cone with the cone point being the vertex in the interior of a 3-cell. It is clear that this map is $c^{\prime}(K, I)$-Lipschitz for some constant $c^{\prime}(K, I)$. Note that it need not be bi-Lipschitz, because it is possible that some tetraheda in $M_{P L}$ may be mapped to nearly flat tetrahedra in $M$.

Proof of Theorem 1.3. Let $M$ be a solid torus with a Riemannian metric as in the statement of the theorem. Let $T$ be the triangulation given by Proposition 5.1. By Theorem 1.1, there is a pre-core curve $C$ that lies in the 2-skeleton of $T$, that intersects the 1-skeleton in only finitely many points and that intersects the interior of each face in at most 10 straight arcs. We make an arbitrarily small perturbation of $C$ to make it into a core curve. So the length of $C$ in $M_{P L}$ is at most $40|T|$, where $|T|$ is the number of tetrahedra. By (2) of Proposition 5.1, $|T|$ is at most $c^{\prime}(K, I) V$. By (1) of Proposition 5.1, there is a Lipschitz homeomorphism $M_{P L} \rightarrow M$ with Lipschitz constant $c^{\prime}(K, I)$. Thus, the length of the image of $C$ in $M$ is at most $40\left(c^{\prime}(K, I)\right)^{2} V$.

\section{EXAMPLES}

In this section, we investigate a family of triangulations of the solid torus. Using them, we will prove the following.

Theorem 6.1. There exists a family of triangulations $T_{i}(i \in \mathbb{N})$ of the solid torus $M$, with the following properties:

1. the number of triangles and squares in any normal meridian disc is at least $c^{\left|T_{i}\right|}$, where $\left|T_{i}\right|$ is the number of tetrahedra in $T_{i}$ and $c$ is the golden ratio $(1+\sqrt{5}) / 2$ 


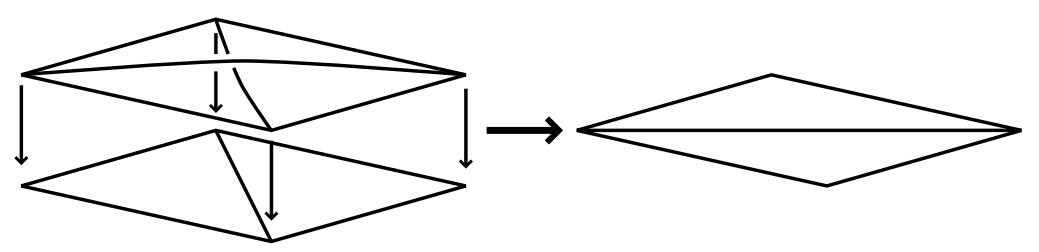

FIGURE 9

2. each pre-core curve in $\partial M$ intersects the 1 -skeleton of $T_{i}$ in at least $c^{\left|T_{i}\right|-1}$ points;

3. there is a pre-core curve that lies in the 2-skeleton of $T_{i}$ and that intersects the 1-skeleton in just one point. Moreover, when $i \geq 1$, this is in fact a core curve.

These examples exhibit the intrinsically exponential nature of normal surface theory. They also show that it would be impossible to prove Theorem 1.1 by using only pre-core curves in $\partial M$.

Proof. Start with a fixed triangulation $T_{0}$ of the solid torus $M$. For the sake of being definite, we use the triangulation with a single tetrahedron. (This is described in 4 for example.) This restricts to a one-vertex triangulation of the boundary torus. The three edges on the boundary have slopes $(1,0),(2,1)$ and $(3,1)$. Here, we are using the standard basis for the first homology of the torus, where $(0,1)$ is a meridian and $(1,0)$ is a longitude.

We will construct the triangulations $T_{i}$ recursively, with each obtained from its predecessor by attaching a tetrahedron onto its boundary, as shown in Figure 9. This has the effect of performing an elementary move on the boundary triangulation. This removes one of the three edges of the boundary triangulation so that the two triangles patch together to form a square, and then inserts the other diagonal of this square.

It is well known that the set of one-vertex triangulations of a torus forms the vertices of a tree. Two vertices are joined by an edge if and only if the corresponding triangulations differ by an elementary move. See Figure 10. Our initial triangulation $T_{0} \cap \partial M$ corresponds to one of these vertices. To obtain $T_{1}$, we perform the elementary move that removes $(1,0)$ and inserts $(5,2)$. Then, to obtain $T_{2}$, we remove $(2,1)$ and insert $(8,3)$. We repeat in this way, following the path in this tree that turns left, then turns right, then turns left, and so on. This gives our sequence of triangulations $T_{i}$ of $M$.

We now compute the slopes of the 1-cells in the boundary torus of $T_{i}$. Let $s_{i}$ be the slope of the 1-cell that is removed when passing from $T_{i}$ to $T_{i+1}$. We orient this slope so that it represents a homology class with non-negative intersection number with the meridian. Then each $s_{i}$ is, in standard homology co-ordinates, $\left(x_{i}, y_{i}\right)$. So, $s_{0}=(1,0)$ and $s_{1}=(2,1)$. It follows from our construction that, for each $i, s_{i+2}=s_{i}+s_{i+1}$. In particular, the integers $y_{i}$ satisfy the Fibonacci relation $y_{i+2}=y_{i}+y_{i+1}$, where $y_{0}=0$ and $y_{1}=1$. Hence,

$$
y_{i}=\frac{1}{\sqrt{5}}\left(\frac{1+\sqrt{5}}{2}\right)^{i}-\frac{1}{\sqrt{5}}\left(\frac{1-\sqrt{5}}{2}\right)^{i} .
$$




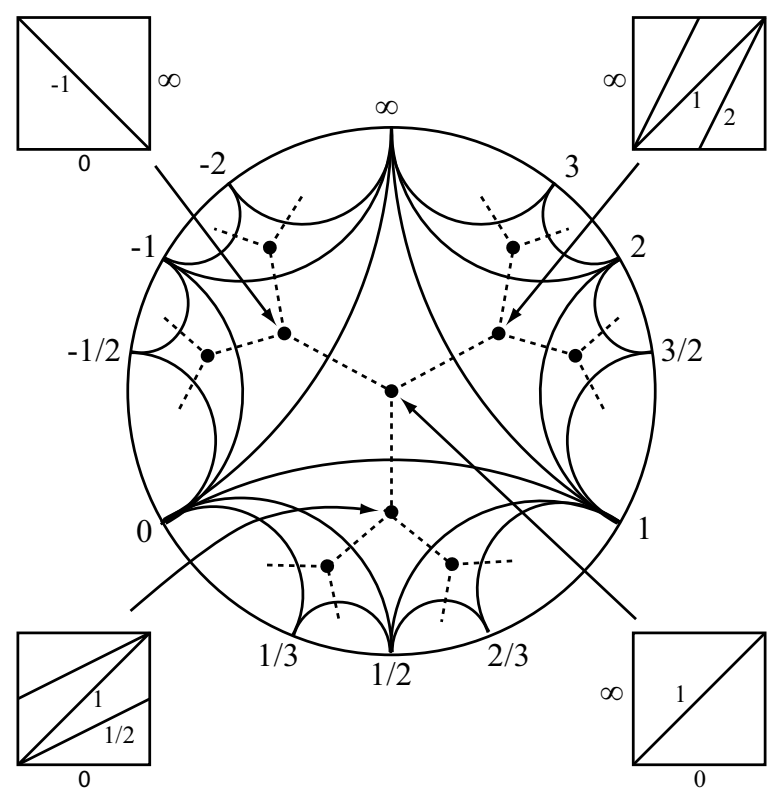

FiguRE 10

The integers $x_{i}$ also satisfy the Fibonacci relation, and since $x_{0}=1=y_{2}$ and $x_{1}=2=y_{3}$, we deduce that $x_{i}=y_{i+2}$ for all $i$.

We want to find a lower bound on the number of triangles and squares of any normal meridian disc in $T_{i}$. Now, $T_{i}$ contains an edge with slope $s_{i+2}$ and each normal triangle or square can intersect this edge at most once. Thus, it suffices to find a lower bound on the intersection number between $s_{i+2}$ and any meridian curve. But this is $x_{i+2}$, which is at least $c^{i+1}=c^{\left|T_{i}\right|}$. This proves (1).

Proving (2) is slightly more tricky because a curve on the boundary of the solid torus with slope $(1, n)$, for any integer $n$, is a pre-core curve. However, the intersection number between $(1, n)$ and $s_{i+2}$ is

$$
\left|n x_{i+2}-y_{i+2}\right|=\left|n x_{i+2}-x_{i}\right|=x_{i+2}\left|n-\left(x_{i} / x_{i+2}\right)\right| .
$$

Now, as $i \rightarrow \infty, x_{i} / x_{i+2} \rightarrow c^{-2}$, which is not an integer. So, there is a uniform lower bound to the difference $\left|n-\left(x_{i} / x_{i+2}\right)\right|$ provided $i$ is sufficiently large, and in fact one can verify that it is always at least $1 / 3$. So, the intersection number between any pre-core curve on $\partial M$ and $s_{i+2}$ is at least $x_{i+2} / 3 \geq c^{i}=c^{\left|T_{i}\right|-1}$, as required.

Finally, note that $T_{0}$ contains a pre-core curve that lies in the 2-skeleton and that intersects the 1-skeleton in just one point. This point lies in the interior of the edge on $\partial M$ with slope $(1,0)$. Thus, this curve becomes a core curve in $T_{1}$ and all subsequent triangulations.

\section{REFERENCES}

[1] Wolfgang Haken, Theorie der Normalflächen (German), Acta Math. 105 (1961), 245-375. MR0141106 (25 \#4519a) 
[2] Joel Hass and Jeffrey C. Lagarias, The number of Reidemeister moves needed for unknotting, J. Amer. Math. Soc. 14 (2001), no. 2, 399-428 (electronic), DOI 10.1090/S0894-0347-01-00358-7. MR:1815217 (2001m:57012)

[3] William Jaco and Ulrich Oertel, An algorithm to decide if a 3-manifold is a Haken manifold, Topology 23 (1984), no. 2, 195-209, DOI 10.1016/0040-9383(84)90039-9. MR7444850 (85j:57014)

[4] William Jaco, Hyam Rubinstein, and Stephan Tillmann, Minimal triangulations for an infinite family of lens spaces, J. Topol. 2 (2009), no. 1, 157-180, DOI 10.1112/jtopol/jtp004. MR2499441(2010b:57016)

[5] Marc Lackenby, Word hyperbolic Dehn surgery, Invent. Math. 140 (2000), no. 2, 243-282, DOI 10.1007/s002220000047. MR1756996 (2001m:57003)

[6] Marc Lackenby, The crossing number of composite knots, J. Topol. 2 (2009), no. 4, 747-768, DOI 10.1112/jtopol/jtp028. MR2574742(2011e:57013)

[7] Marc Lackenby, The crossing number of satellite knots, arxiv:1106.3095.

[8] Sergei Matveev, Algorithmic topology and classification of 3-manifolds, Algorithms and Computation in Mathematics, vol. 9, Springer-Verlag, Berlin, 2003. MR.1997069 (2004i:57026)

Mathematical Institute, University of Oxford, 24-29 St Giles', Oxford OX1 3LB, United Kingdom 